\section{Micellar interpolyelectrolyte complexes}

\author{
Dmitry V. Pergushov, ${ }^{a}$ Axel H. E. Müller ${ }^{b}$ and Felix H. Schacher*c \\ Received 10th April 2012 \\ DOI: $10.1039 / \mathrm{c} 2 \mathrm{cs} 35135 \mathrm{~h}$
}

Interpolyelectrolyte complexes (IPECs) are typically formed when two polyelectrolytes of opposite charge are mixed together in solution. We present an overview of different strategies for the preparation of micellar IPECs, i.e., structures where such IPEC domains form the core or the shell of micelles. In addition, vesicular architectures are considered, where the IPEC domain forms a membrane layer. One intriguing feature of IPECs is that their formation can be directed, their stability towards changes in $\mathrm{pH}$ or ionic strength can (to a certain extent) be predicted, and their size can be controlled. Especially the use of ionic/non-ionic block copolymers offers unique potential for the preparation of well-defined and sophisticated nanostructured materials. We also discuss possible applications, especially in the field of life sciences, including biocompatibility, the controlled uptake/release of guest substances, the immobilization of enzymes, or the controlled formation of inorganic/organic hybrid materials.

\section{Introduction}

Interpolyelectrolyte complexes (IPECs) are typically formed when two oppositely charged polyelectrolytes are mixed in solution.

${ }^{a}$ Department of Chemistry, M.V. Lomonosov Moscow State University, Vorob'evy Gory, 119991 Moscow, Russia

${ }^{b}$ Makromolekulare Chemie II and Bayreuther Zentrum für Kolloide und Grenzflächen, Universität Bayreuth, D-95440 Bayreuth, Germany

${ }^{c}$ Institut für Organische Chemie und Makromolekulare Chemie and Jena Center for Soft Matter (JCSM), Friedrich-Schiller-Universität Jena, D-07743 Jena, Germany. E-mail: felix.schacher@uni-jena.de
This represents a facile and straightforward way to direct co-assembly processes of macromolecules, depending on a variety of parameters such as the charge-to-charge stoichiometry, the solution conditions, or the molecular weight of the involved building blocks. Further, it offers unique control over both the properties and the morphology of the resulting macromolecular architectures. This has been extensively shown for IPECs since their discovery in solution in $1949^{1,2}$ and also for similar processes occurring at surfaces after the discovery of the layer-by-layer technique in $1997 .^{3}$ During the last few years, this has been further extended to several

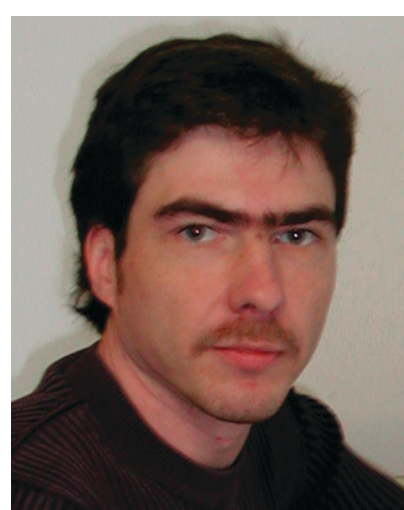

Dmitry V. Pergushov
Dmitry V. Pergushov graduated from M.V. Lomonosov Moscow State University in 1993. In 1996, he obtained his PhD degree at the Department of Chemistry at the same university, working on interpolyelectrolyte complexes with Prof. Victor A. Kabanov and Prof. Alexander B. Zezin. Since 2001, he has been a senior researcher in the same department. He was a visiting researcher at the Leibniz Institute for Polymer Research in Dresden, Germany, and the University of Bayreuth, Germany. He received a fellowship within the framework of the NATO Science Program in 2001 and a grant of the President of the Russian Federation for Young Russian Scientists in 2004. His research interests are concentrated on co-assembled macromolecular architectures with a particular focus on interpolyelectrolyte complexes.

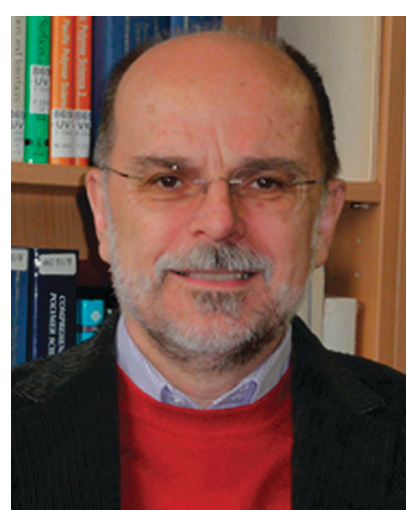

Axel H. E. Müller
Axel H. E. Müller obtained his PhD in 1977 at the Johannes Gutenberg University in Mainz, Germany, working on the kinetics of anionic polymerization with G. V. Schulz. Since 1999 he has been a professor and chair of Macromolecular Chemistry at the University of Bayreuth. In 2004 he received the IUPAC MACRO Distinguished Polymer Scientist Award and in 2012 he was awarded the Hermann Staudinger Award of the German Chemical Society (GDCh). Further, he is a Senior Editor of the journal Polymer. His research interests focus on the design of welldefined polymer structures by controlled/living polymerization techniques and on self-organized nanostructures and hybrids obtained from them. He co-edited five books and published over 400 research papers. 
more sophisticated systems, in particular double hydrophilic or amphiphilic diblock copolymers comprising ionic and non-ionic segments, polyampholytic triblock terpolymers, branched polyelectrolytes (polyelectrolyte stars and cylindrical polyelectrolyte brushes), or ionic dendrimers. In that way, different macromolecular architectures have been created, including micelles, micellar networks, and zipper brushes. ${ }^{4,5}$ We think that the predictability of the generated structures as well as the possibility to induce dynamics via, e.g., variations in $\mathrm{pH}$ or ionic strength of the surrounding microenvironment render the use of interpolyelectrolyte complexation a promising approach for the fabrication of advanced polymeric reagents. Such materials might be interesting for applications related to sensing, structuring, or the controlled uptake and release of guest substances, especially in the case of biologically active examples.

\section{Background}

Polyelectrolytes are a class of water-soluble macromolecules carrying high amounts of charges. In general, such materials can be divided into either weak (annealed) or strong (quenched) polycations and polyanions. Strong polyelectrolytes are permanently charged, regardless of the $\mathrm{pH}$ in aqueous media and prominent examples are poly(sodium styrene sulfonate) (PSSNa, polyanion) ${ }^{6}$ or poly(diallyldimethylammonium chloride) (PDADMAC, polycation). ${ }^{7}$ For weak polyelectrolytes, the situation is different: here, the charge density and, hence, the chain conformation and the solubility depend on the solution $\mathrm{pH}$, as shown for poly(acrylic acid) (PAA) and poly(methacrylic acid) (PMAA), polyanions) ${ }^{8}$ or poly(2-(dimethylamino)ethyl methacrylate) (PDMAEMA, polycation). ${ }^{9}, 10$

The synthesis of polyelectrolytes can be achieved by a variety of polymerization techniques, including radical, ionic, or ring-opening methodologies. Especially in the case of controlled/ living polymerization approaches, continuous improvements

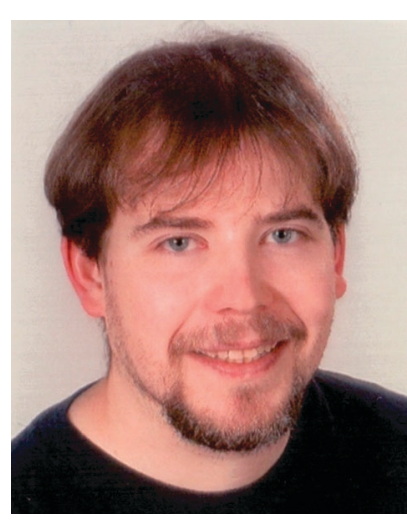

Felix H. Schacher
Felix H. Schacher was born in Lichtenfels, Germany, in 1980. After finishing his graduate studies in chemistry at the University of Bayreuth, Germany, in 2006, he completed his doctoral work under supervision by Prof. Axel H. E. Müller in 2009. His thesis focused on living anionic polymerization techniques and functional nanostructured systems based on block co- and terpolymers in the bulk and in solution. Afterwards, he joined the group of Prof. Ian Manners as a DAAD postdoctoral fellow, working on the synthesis and self-assembly of organometallic block copolymers. Since 2010, he is a junior professor at the Friedrich-SchillerUniversity Jena, Germany. His research interests are polymer synthesis, self-assembly of polymer-based materials, and different characterization techniques related to block copolymer nanostructures. regarding monomer diversity or purification prerequisites allow for a more and more precise positioning and manipulation of charged segments within polymeric materials. A detailed description would be beyond the scope of this review, but recent trends in the synthesis of polyelectrolytes focus on the introduction of additional functionality to the materials (e.g., charges in combination with building blocks carrying hydrogen bonding motifs ${ }^{11}$ ) or on the flexibility of the polymeric backbone. ${ }^{12}$ Also, the controlled polymerization of monomers exhibiting multiple charges per repeating unit ${ }^{13}$ or ionic liquid-based building blocks (poly(ionic liquids), PILs) as a new class of polyelectrolytes is currently being intensively investigated. ${ }^{14,15}$ Other than cationic or anionic polyelectrolytes, polyampholytes contain both positive and negative charges, which can be randomly distributed or arranged blockwise along the polymeric backbone. ${ }^{16}$ In the case of polyzwitterions, each monomer unit carries at least one positive and one negative charge. ${ }^{17}$

If two oppositely charged homopolyelectrolytes are mixed, interpolyelectrolyte complexes (IPECs) are formed spontaneously (Fig. 1) due to cooperative electrostatic interactions. Such structures were proposed to consist of both areas with a rather ordered chain packing ("ladder-like" sequences) ${ }^{18}$ and also disordered ("scrambled egg") domains. ${ }^{19}$ The main driving force for complex formation in aqueous media is the release of the low molecular weight counterions which were previously associated with the charged groups on the macromolecules and the resulting gain in entropy for the system. Nevertheless, IPECs can be additionally stabilized by hydrogen bonding or hydrophobic interactions, ${ }^{20}$ though such systems will not be the focus of this review.

The general observation that aqueous mixtures of oppositely charged colloidal systems (natural polymers with rather low charge densities) undergo phase separation (coagulation or coacervation) close to isoelectric conditions has already been made in the early 20th century. ${ }^{21,22}$ First investigations on complex formation between oppositely charged synthetic homopolyelectrolytes with high charge densities were performed in 1949 by Fuoss et al. ${ }^{1}$ (flocculent precipitates) and later in 1961 by Michaels and coworkers (polyanion-polycation complexes), ${ }^{2}$ followed by systematic investigations during the next decades, including pioneering work by the groups of Tsuchida ${ }^{23,24}$ and Kabanov and Zezin. ${ }^{18,25}$ During these decades, different nomenclatures have been used in the literature for such macromolecular co-assemblies:

- Interpolyelectrolyte complex (IPEC), a specific term for the complex formation between two synthetic polyelectrolytes of opposite charge; first appearance $1989 .{ }^{26}$

- Block ionomer complex (BIC), another term specifically used for the complex formation between ionic/non-ionic block copolymers with one charged and one neutral segment and polyelectrolytes of opposite charge; first appearance in $1996 .^{27}$

- Polyion complex (PIC), also often used for the complexation of deoxyribonucleic acid (DNA) or peptide sequences with polyelectrolytes; first appearance $1969 .{ }^{28}$

- Polyplex, a term mainly used for complexes between polyelectrolytes and DNA.

- Complex coacervate (CC), used since 1929 first for the description of mixtures of oppositely charged natural polymers 


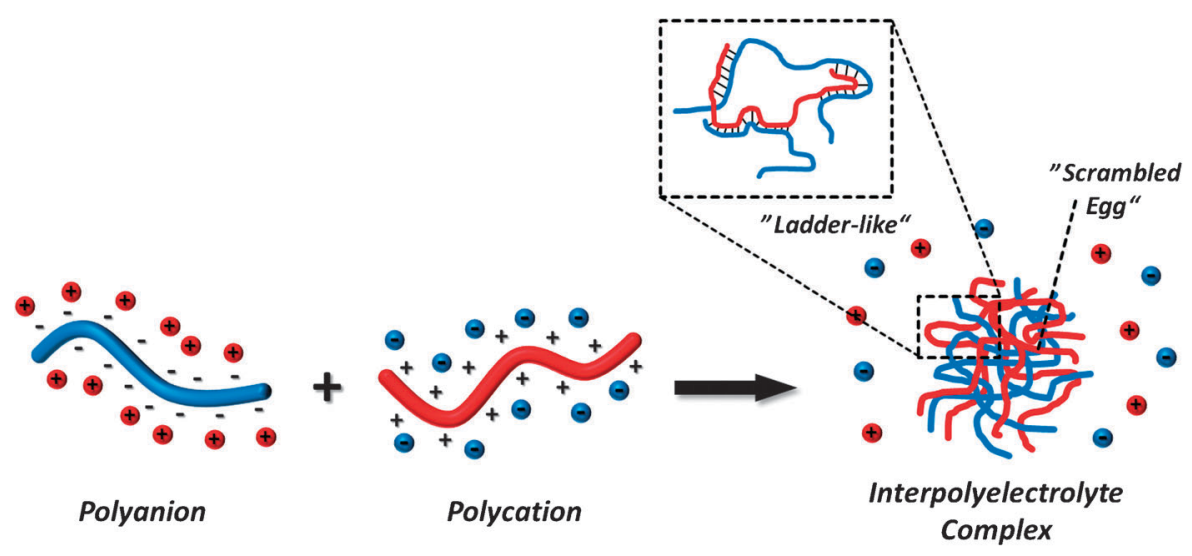

Fig. 1 Schematic depiction of interpolyelectrolyte complex (IPEC) formation between polycations and polyanions with a concomitant release of the low molecular weight counterions.

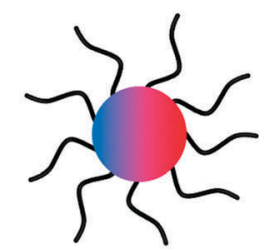

IPEC Domain:

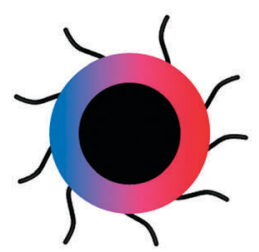

Shell

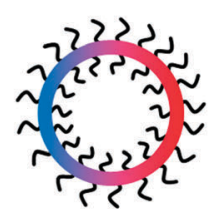

Membrane

Fig. 2 Schematic depiction of micellar/vesicular architectures involving interpolyelectrolyte complex (IPEC) domains: structures where the IPEC domain forms the core (left), the shell (middle), or the membrane layer (right).

with rather low charge densities, i.e., gelatin and gum arabic, ${ }^{22}$ introduced in 1998 for the electrostatic co-assembly of oppositely charged synthetic polyelectrolytes. ${ }^{29}$

Throughout this tutorial review, we will use the term interpolyelectrolyte complex (IPEC) where appropriate.

Important general applications for IPECs have been their use as effective and environmentally friendly binders of soil and sand that were applied, for example, in Chernobyl to prevent spreading of radionuclides induced by water and/or wind erosion, ${ }^{25,30}$ in the construction of polyelectrolyte multilayers on surfaces $^{3}$ and colloids ${ }^{31}$ via the layer-by-layer technique, or as membranes for pervaporation, nanofiltration, and fuel cell applications. ${ }^{32}$ It could further be demonstrated that such systems are capable of molecular recognition. ${ }^{33,34}$ Other approaches focused on the complexation of DNA or ribonucleic acid (RNA) into IPECs for non-viral gene transfection ${ }^{35-37}$ or the incorporation of selected enzymes (trypsin, $\alpha$-chymotrypsin, penicillin amidase, etc.) via chemical or physical immobilization, ${ }^{26,38}$ which even exhibited activities close to those of the "free" enzyme. In addition, IPECs are characterized by a high permeability for water, which renders these structures interesting for medicinal purposes. Kabanov, Zezin, and coworkers have already shown that IPECs show high resistance against thromboformation. ${ }^{39}$ Nowadays, IPEC-based systems are already on their way to medically relevant delivery systems. ${ }^{40}$

\section{Scope of this review}

The main focus of this review is on micellar IPECs, both in aqueous and organic media. We primarily describe examples, where IPEC domains form either the core or the shell (continuous or patchy) of micellar aggregates. In addition, vesicular structures where the membrane layer is formed via the complexation of oppositely charged polymers are described as well. A schematic depiction of the main micellar/vesicular architectures comprising IPEC domains is shown in Fig. 2.

Further, we included selected examples of multi-layered architectures, e.g., the formation of polyelectrolyte multilayer capsules or first approaches where more than one IPEC layer is generated within micellar structures. For IPECs involving branched polyelectrolytes (star-shaped polymers or cylindrical polymer brushes) the reader is referred to the recent literature. ${ }^{5}$

\section{Interpolyelectrolyte complexes - formation and stability}

If aqueous solutions of oppositely charged polyelectrolytes are mixed, interpolyelectrolyte complexes (IPECs) are formed immediately. The kinetics of complex formation have been first investigated by Kabanov and Zezin and stop flow measurements have shown that this process takes place in less than 5 milliseconds. ${ }^{41}$ Any low molecular weight counterions, which are initially located near the polymeric backbone of the involved macroions (counterion condensation), are released and the resulting gain in entropy for the whole system is the main driving force for this process. It is worth noting that the formation of IPECs is not limited to aqueous media: the mixing of two different (complementary) polyelectrolytesurfactant complexes in chloroform resulted in the formation of IPECs and release of the surfactant counterions. ${ }^{42}$ 
For interpolyelectrolyte complexation, a very important parameter is the actual charge-to-charge stoichiometry, more precisely the overall ratio of positive to negative charges of the oppositely charged polyelectrolytes involved. This ratio is defined as $Z$ with the subscript $(+/-$ or $-/+)$ denoting the charge ratio. Analogously, the charge-to-charge stoichiometry of the formed IPECs is characterized by the ratio of positive to negative charges of the incorporated macroions $(\varphi$, also here, any subscripts denote the charge ratio). In general, $\varphi$ might be different from $Z$ of the original mixture. ${ }^{18,25}$ Stoichiometric IPECs $(\varphi=1)$ are sufficiently hydrophobic due to the mutual screening of the charges and precipitate from aqueous solution, although a certain swelling (swelling degrees of 1-10 have been found for IPECs based on slightly crosslinked polyelectrolytes of $1: 1$ stoichiometry ${ }^{25}$ due to the incorporation of water molecules cannot be excluded. If non-stoichiometric $(Z \neq 1)$ mixtures are prepared, overcharging effects due to an excess of either polycation or polyanion can be observed, also depending on the molecular weight of the respective polyelectrolyte added in excess. ${ }^{18,25,41,43-45}$ This has been studied using fluorescence spectroscopy using labeled polyelectrolytes. ${ }^{18,41,44,45}$

Two types of equilibria have to be considered in order to describe interpolyelectrolyte complexation (Fig. 3): type (I) describes the reversible association of oppositely charged macromolecules $\mathrm{M}_{1}$ and $\mathrm{M}_{2}$, which can be regarded as single reactive species. Here, the overall number of electrostatic interactions (interpolymer salt bonds between $\mathrm{M}_{1}$ and $\mathrm{M}_{2}$ ) is in general not important. In contrast, for equilibrium (II) $\mathbf{M}_{1}$ and $\mathrm{M}_{2}$ are considered as polyfunctional reactive species and here the complexation is described as a reversible reaction between $m_{1}$ and $m_{2}$ individual charged groups $\left(m_{1}\right.$ and $m_{2}$ denote the degrees of polymerization for the involved polyelectrolytes $\mathrm{M}_{1}$ and $\mathrm{M}_{2}$ ). In this case, pairs of oppositely charged groups of $m_{1}$ and $m_{2}$ may or may not form interpolymer salt bonds, whereas the latter are to be discriminated. Therefore, a detailed analysis of electrostatically driven macromolecular co-assembly should be based on a combined consideration of both described equilibria (I + II).

A thorough study of equilibria (I) and (II) for interpolyelectrolyte complexation between PDMAEMA of high molecular weight and oligo- or polyphosphates (OPs or PPs) with different degrees of polymerization was carried out using analytical ultracentrifugation (AUC) and potentiometric titration. ${ }^{46}$ The combination of these techniques allowed for the determination of the fraction $\Phi$ of PP chains, which were coupled to PDMAE$\mathrm{MA}$, and the conversion $\theta$ for the formation of interpolymer salt bonds at different $\mathrm{pH}$-values. $\Phi$ characterizes equilibrium (I) while $\theta$ describes equilibrium (II). It could be demonstrated that PPs of rather high molecular weights were almost completely bound to PDMAEMA $(\Phi \cong 1)$ already at rather low degrees of conversion $(\theta \approx 0.1)$. Further formation of interpolymer salt bonds occurs within the now-generated IPEC domains. Here, a thorough analysis of IPEC formation can only be achieved via consideration of equilibrium (II). In contrast, interaction between OPs and PDMAEMA resulted in a gradual increase of $\theta$ with rising $\Phi$ : thus, rather high degrees of conversion concerning interpolymer salt bonds already occur at rather low values of $\Phi$. In this case, IPEC formation (in the limit) can be described by equilibrium (I). It is worth noting that for a fixed value of $\theta$ increasing degrees of polymerization for the OPs/PPs lead to higher chain fractions coupled to PDMAEMA while for a fixed value of $\Phi$ the conversion $\theta$ decreases with increasing lengths of OPs/PPs.

Another important parameter, besides the charge-to-charge stoichiometry of IPECs $(\varphi)$, is the length ratio of the charged segments. It has been shown for mixtures of bis-hydrophilic diblock copolymers with one charged (cationic or anionic) and another uncharged segment (poly(ethylene oxide), PEO) that the resulting IPECs are more well defined if the lengths of the oppositely charged blocks match. ${ }^{33}$ Moreover, light scattering experiments also indicated a preferred co-assembly of block copolymers with matching polyelectrolyte segment lengths when combinations of different degrees of polymerization were used. Further studies by the same authors later revealed that the length of the charged segments in PEO-block$\operatorname{poly}(\alpha, \beta$-aspartic acid) (PEO- $b$-PAsp) has a pronounced influence on the aggregation number and, hence, on the density of PEO chains at the core-corona interface for the IPEC micelles formed upon mixing with oppositely charged poly(lysine) (PLys). ${ }^{47}$

The presence of salt (e.g., $\mathrm{NaCl})$ drastically affects the characteristics of IPECs. ${ }^{18,25,48,50,51}$ This has been clearly demonstrated for water-soluble non-stoichiometric examples. ${ }^{18,25,48,50}$ At low salt levels, the complexation leads to the formation of rather small particles. Increasing ionic strength can induce the formation of aggregated IPEC species and even cause macroscopic phase separation, leading to a precipitate of an insoluble nearly stoichiometric $(\varphi \cong 1$ ) IPEC while the prevailing

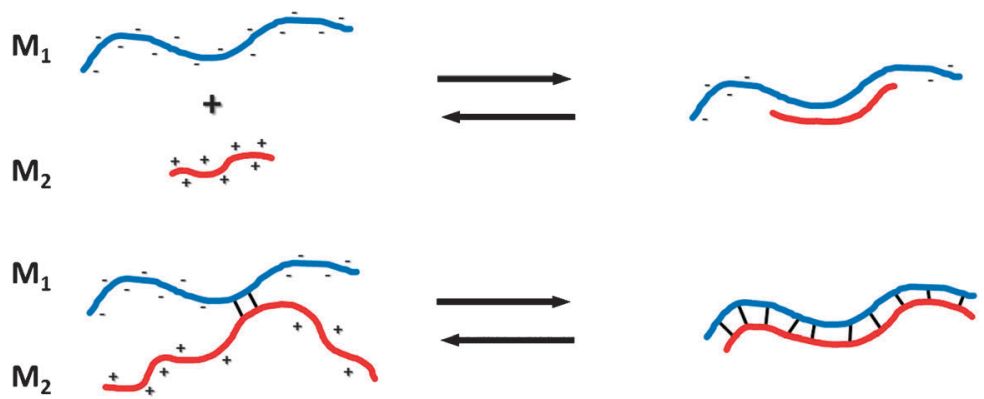

(I)

Fig. 3 Two separate equilibria describing interpolyelectrolyte complex (IPEC) formation; reversible association of oppositely charged macromolecules $\mathrm{M}_{1}$ and $\mathrm{M}_{2}$ (I) and the buildup of interpolymer salt bonds between two polyfunctional reactive species with the degrees of polymerization $m_{1}$ and $m_{2}$ (II). 
supernatant solution contains either non-stoichiometric $(\varphi \neq 1)$ IPECs or in some cases a pure host (excess) polyelectrolyte. At still higher salt concentrations, all polyelectrolyte chains are completely screened, thus separated, and do not interact. Therefore, no complex formation is observed. The specific salt concentrations for the abovementioned regimes are influenced by a number of factors, among which are the nature of the ionic groups of the constituting polyelectrolytes, ${ }^{49,51}$ the low molecular weight counter ions of the added salts, ${ }^{25,50,51}$ the charge-to-charge ratio of the involved polyelectrolytes, ${ }^{25,49}$ and their degrees of polymerization. ${ }^{18,25,48}$ In addition to the salt concentration, in the case of weak polyelectrolytes, the solution $\mathrm{pH}$ strongly influences the amount of charges and therefore also the extent of interpolyelectrolyte complexation occurring.

The observed salt dependence on IPEC characteristics can also be exploited after the complex has been formed, thus rendering materials that are very sensitive to changes in their surrounding environment. Regarding micellar IPECs with either the IPEC core or shell domains, this enables polyion exchange reactions above a certain ionic strength and leads to dynamic systems. Such processes, i.e., interpolyelectrolyte exchange/substitution and addition reactions, could be monitored using polycation-quenchers in combination with fluorescently labeled polyanions. ${ }^{18,41,52,53}$ Kinetic investigations revealed that the rate of polyion exchange is also crucially dependent on the structure of the IPECs: Kabanov and coauthors showed that these processes occur faster in the case of non-stoichiometric micellar IPECs formed between PEO- $b$ PMAA and quaternized poly(4-vinylpyridine) (P4VPq) when compared to common PMAA/P4VPq systems. ${ }^{53}$ This was explained through greater electrostatic repulsion experienced by the free PMAA segments when compared to IPECs with a shielding PEO corona. More surprising was the fact that the diffusion of polyions into the IPEC core of these structures was not hindered by the PEO segments. At the same time, a considerable decrease of the polyion exchange rate for non-stoichiometric micellar IPECs formed by polystyreneblock-PMAA (PS-b-PMAA) and P4VPq was demonstrated. ${ }^{52}$

In summary, both the stability and the structure of IPECs are influenced by a number of factors: obvious parameters are the charge-to-charge stoichiometry of the involved polyelectrolytes, their respective concentrations, degrees of polymerizations, the length ratio of the charged segments, and the charge densities. This is further affected by the surrounding medium, most importantly the $\mathrm{pH}$ and the ionic strength. However, also the nature of the ionic groups of the polyelectrolytes, the geometry, charge, and nature of the low molecular weight counterions, or the presence of uncharged water-soluble segments as shown for, e.g., bis-hydrophilic PEO- $b$-PMAA block copolymers, can play an important role.

\section{Micellar interpolyelectrolyte complexes with an IPEC core}

The mixing of stoichiometric amounts $(Z=1)$ of oppositely charged homopolyelectrolytes in aqueous media results in the formation of sufficiently hydrophobic IPECs. The precipitation from aqueous solution can be effectively prevented if at least one of the polyelectrolytes is covalently attached to another hydrophilic non-ionic segment, which itself does not take part in complex formation (e.g., bis-hydrophilic ionic/ non-ionic diblock copolymers). In that way, micellar structures are formed where the IPEC builds up the core. The aggregates are solubilized by a corona consisting of the additional hydrophilic segments. Different scenarios for the formation of such core-corona IPEC particles can be described (Fig. 4).

\section{$\mathbf{A B}+\mathbf{C}$}

If bis-hydrophilic diblock copolymers (AB) with a non-ionic (A) and an ionic (B) segment are mixed in aqueous solution with an oppositely charged linear homopolyelectrolyte (C), micellar IPECs with a core consisting of complexed B and C

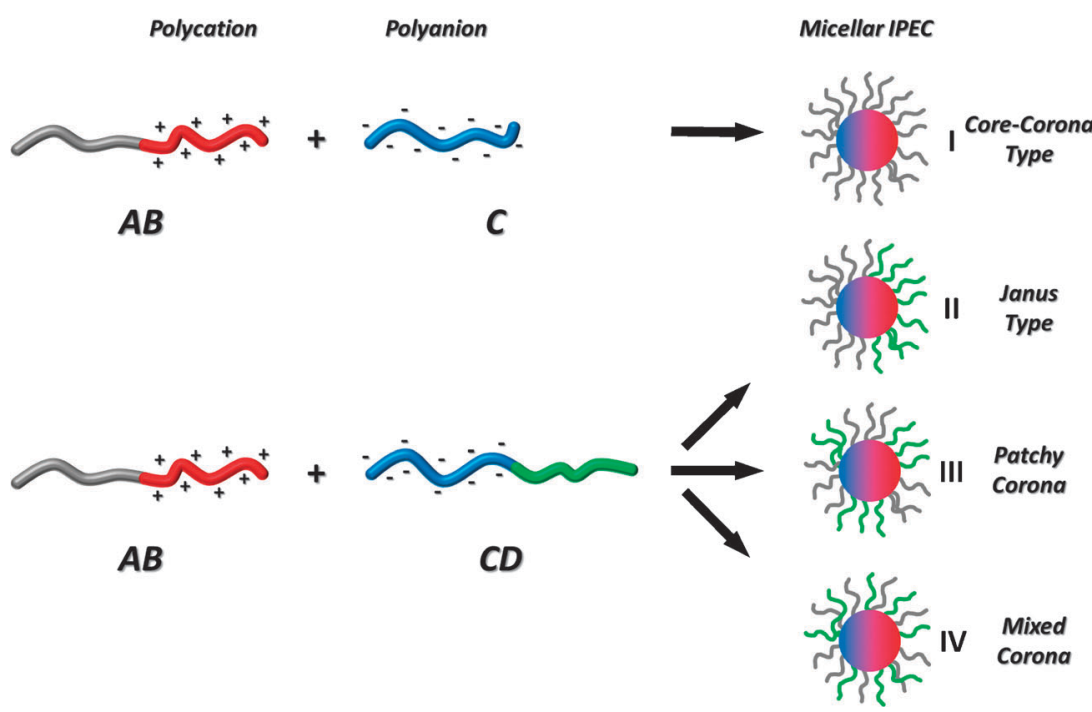

Fig. 4 Schematic depiction of interpolyelectrolyte complexation at $Z=1$, resulting in micellar structures with an IPEC core (I) between neutralblock-cationic $\mathrm{AB}$ diblock copolymers and linear homopolyelectrolytes of opposite charge $(\mathrm{C})$ leading to core-corona micelles with a corona formed by A; and (II) between neutral-block-cationic AB and neutral-block-anionic CD diblock copolymers, resulting in micellar IPECs with a Janus (II), patchy (III), or mixed corona (IV) of A and D segments. 

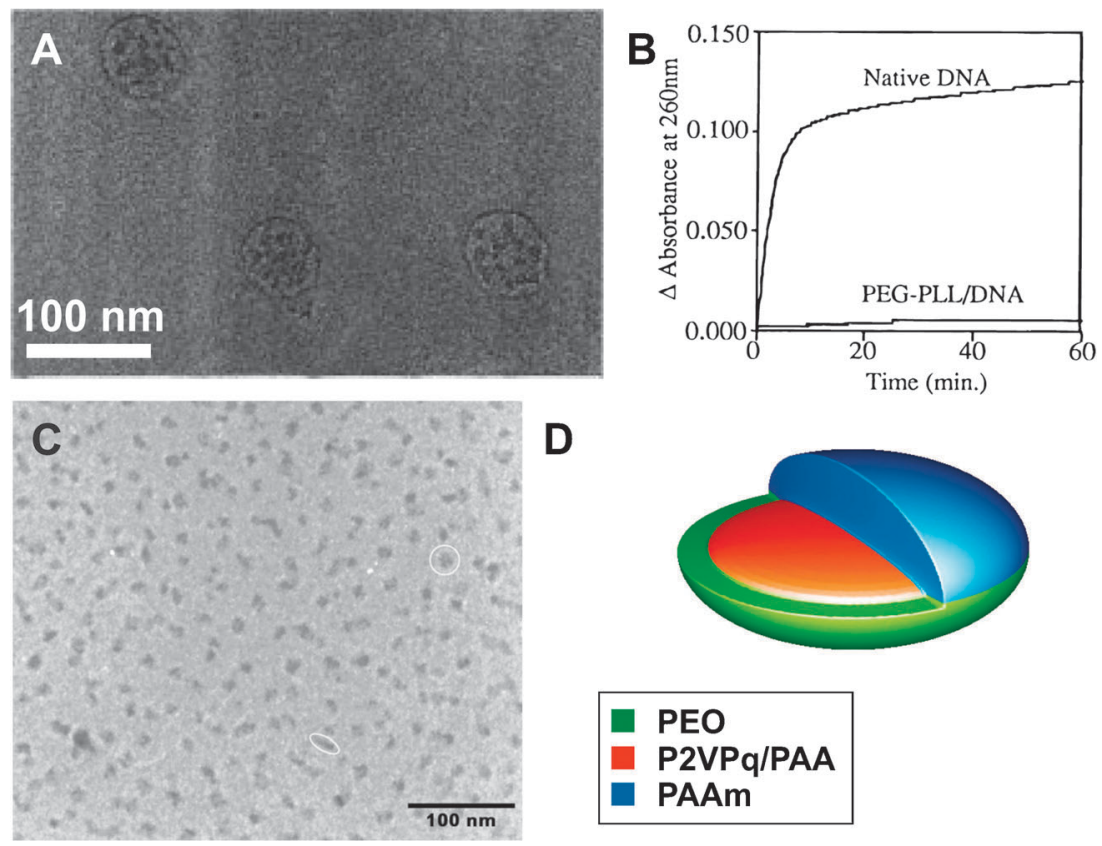

D
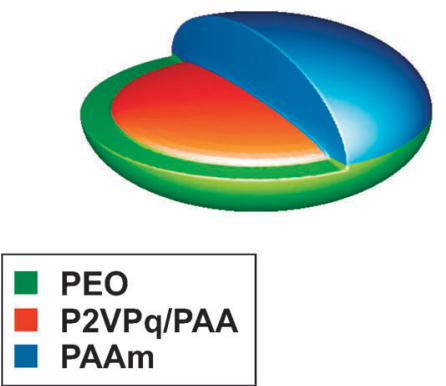

Fig. 5 Cryo-TEM micrograph of IPEC micelles formed by mixing PDMAEMA- $b$-PGMA and PAA (A, reprinted with permission from ref. 29. Copyright 1998 American Chemical Society); increased stability of DNA against enzymatic degradation via encapsulation within a micellar IPEC core (B, reprinted with permission from ref. 35. Copyright 1997 American Chemical Society); cryo-TEM micrograph of Janus-type micellar IPECs formed by complexation of PEO- $b$-P2VPq with PAA-b-PAAm diblock copolymers (C); schematic depiction of the so-called "double-faced micelles" (D, reprinted with permission from ref. 58. Copyright 2006 Wiley Interscience).

segments and a corona of $\mathrm{A}$ are formed. If the chain length of the A segments is sufficient, the resulting core-corona structures can be well soluble. The scheme in Fig. 4(I) shows an example where the charged $\mathrm{B}$ segment is a polycation and IPEC formation occurs with oppositely charged linear polyanions (C) but, of course, other combinations can be used as well (Fig. 5).

Such core-corona structures have been first demonstrated for poly(sodium methacrylate)-block-poly(ethylene oxide) (PMANa- $b$-PEO) diblock copolymers after mixing with linear poly ( $N$-ethyl-4-vinylpyridinium bromide) (P4VPq) polycations. ${ }^{27}$ The resulting micellar IPECs exhibited a PEO corona and were shown to be both sensitive to the ionic strength of the surrounding media as well as capable of taking part in polyion exchange reactions. In the latter case, the polyion exchange rate was found to increase in the order IPEC $<$ core-corona structures with an IPEC core $<$ core-corona structures with a crosslinked IPEC core. ${ }^{53}$ Kataoka et al. later demonstrated the versatility of this approach for the stabilization and protection of DNA segments: the mixing of PEO- $b$-poly(L-lysine) (PEO$b$-PLL) diblock copolymers with cationic PLL segments and negatively charged DNA samples leads to the formation of micellar IPECs with a PLL/DNA core surrounded by a PEO corona (Fig. 5B) ${ }^{35}$ The presence of the PEO corona drastically increased the resistance of the DNA segments against enzymatic degradation, while still enabling the substitution of DNA by a synthetic linear polyanion, poly(aspartic acid) (PAsp). The stability of such core-corona structures could be further enhanced by, e.g., crosslinking of the IPEC core using disulfide bonds, as shown by the same group for micellar IPECs formed from PEO- $b$-PLL and PAsp. ${ }^{54}$ Cohen Stuart and coworkers reported on micellar IPECs from
PDMAEMA-block-poly(glyceryl methacrylate) (PDMAEMA$b$-PGMA) and PAA (Fig. 5A). ${ }^{29}$ Here, the term complex coacervate core has been used and, later, different combinations of oppositely charged $\mathrm{AB}+\mathrm{C}$ or $\mathrm{AB}+\mathrm{CD}$ (block)polyelectrolytes have been mixed (see also the following paragraphs on systems with a mixed or Janus corona).

\section{$\mathbf{A B}+\mathbf{C D}$}

When two different bis-hydrophilic block copolymers are mixed (AB with a non-ionic (A) and a cationic segment (B) and $\mathrm{CD}$ with an anionic $(\mathrm{C})$ and another uncharged segment (D)), again a micellar core consisting of an IPEC domain between $\mathrm{B}$ and $\mathrm{C}$ is formed but this time the corona of the structure contains both $\mathrm{A}$ and $\mathrm{D}$ chains. If the blocks $\mathrm{A}$ and $\mathrm{D}$ are identical, core-corona IPECs with a homogeneous corona can be expected (like those described in the previous paragraph). This has been demonstrated for pairs of oppositely charged PEO- $b$-PLL and PEO- $b$-PAsp block copolymers. ${ }^{55}$ The same authors were able to show in a later study that the polydispersity of the so-formed micellar IPECs decreases if pairs of block copolymers are used where the charged segments match in length. ${ }^{33}$ For this, they compared a series of block copolymers with degrees of polymerization of 18 and 78 for either PLL or PAsp and studied IPECs where the coreforming segments are of similar $(18+18)$ or different lengths $(18+78,78+18)$. This pronounced "chain length recognition" was explained through a superior packing of the core-forming segments, thereby generating a well-defined interface between the PEO corona and the IPEC domains. Such core-corona IPECs were also studied by Gohy and coworkers using a combination of PEO-b-P2VP and PEO- $b$-PMAA block copolymers 
at different $\mathrm{pH}$-values. ${ }^{56}$ Because in both cases the charged segments comprise a weak polyelectrolyte, IPEC formation was only detected within a limited $\mathrm{pH}$-range.

If the corona-forming blocks A and D are not identical, different scenarios are possible (Fig. 4II-IV): depending on the mutual incompatibility of the two segments, a Janus, patchy, or mixed corona can be formed. However, the differences between a mixed corona and patches might be extremely difficult to resolve, depending on the system investigated. An excellent recent review on such systems has been published by Voets et al. ${ }^{57}$ and we will just highlight two selected examples. Janus structures (II) have been shown by Cohen Stuart and coworkers: they described IPECs with a micellar IPEC core and a phase-separated corona with a PEO and a poly(acryl amide) (PAAm) hemisphere by mixing PEO- $b-\mathrm{P} 2 \mathrm{VPq}$ and PAA- $b$-PAAm block copolymers in appropriate ratios (Fig. 5C and D). ${ }^{58}$ The micellar IPECs exhibited a rather ellipsoidal shape and their Janus character was confirmed by ${ }^{1}$ H-NOESY NMR spectroscopy, showing the absence of cross-correlation signals between the corona-forming blocks, PEO, and PAAm. In another study, micellar IPECs with a (at least partially) mixed corona (IV) were obtained: the mixing of PEO- $b$-P2VPq and poly(acrylic acid)-block-poly( $N$-isopropyl acrylamide) (PAA- $b$-PNIPAAm) leads to micelles with an IPEC core of P2VPq/PAA and a corona build-up from both PEO and PNIPAAm. ${ }^{59}$ Moreover, this system was capable of undergoing structural changes upon heating due to the presence of temperature-responsive PNIPAAm segments. At elevated temperatures, it transformed to a core-shell-corona architecture (see also Fig. 6III), consisting of a collapsed PNIPAAm core, an IPEC shell, and a PEO corona.

There are also other examples where IPECs form the core of micellar structures: Armes and coworkers reported on triple-hydrophilic triblock terpolymers of PEO-block-poly(2-(diethylamino)ethyl methacrylate)-block-poly(2-(succinyloxy)ethyl methacrylate) (PEO- $b$-PDEAEMA- $b$-PSEMA). Depending on the solution $\mathrm{pH}$, different micellar structures with either a hydrophobic PDEAEMA core $(\mathrm{pH}>9$ ), a core consisting of both PEO and PSEMA segments and stabilized via hydrogen bonding $(\mathrm{pH}<4)$, or an IPEC core made up of coupled PDEAEMA/PSEMA segments $(4<\mathrm{pH}<9)$ could be observed. ${ }^{60}$ Here, in contrast to the other examples on IPEC cores, the complexation occurs between covalently bound segments within one single polymer chain, that is, intramolecular IPECs might be also generated. In another recent example, ABC miktoarm star copolymers with PEO, PMAA, and PDEAEMA arms have been prepared and, also here, $\mathrm{pH}$-dependent assembly into micelles with (at an intermediate pH-regime) an IPEC core was observed. ${ }^{61}$ As one example for non-spherical systems exhibiting an IPEC core, Yan and coworkers demonstrated the formation of spherocylindrical micellar IPECs from anionically charged supramolecular coordination polymers and PEO- $b$-P2 VPq diblock copolymers, where the morphology of the structures (spherical or cylindrical) was dependent on the $Z$-value of the overall system. ${ }^{62}$

\section{Micellar interpolyelectrolyte complexes with an IPEC shell}

Amphiphilic diblock copolymers form micelles of different morphology in aqueous media where the hydrophobic segments form the core and the corona is built up by the hydrophilic blocks. Depending on the volume fractions of the constituting segments (and on other factors as well), spherical, cylindrical, or even vesicular structures can be obtained. ${ }^{63}$ If the hydrophilic block is a polyelectrolyte, the micelles exhibit a charged corona, enabling its use as a template and the buildup of further layers by the addition of oppositely charged polyelectrolytes. This then leads to the formation of a hydrophobic IPEC shell, surrounding the micellar core (Fig. 6). If a homopolyelectrolyte (C) is used for IPEC formation (Fig. 6I), the so-formed core-shell-corona structures are stable until a threshold $Z$-ratio is reached. Below this value, the excess of corona-forming charged B segments (here, of anionic charge) is

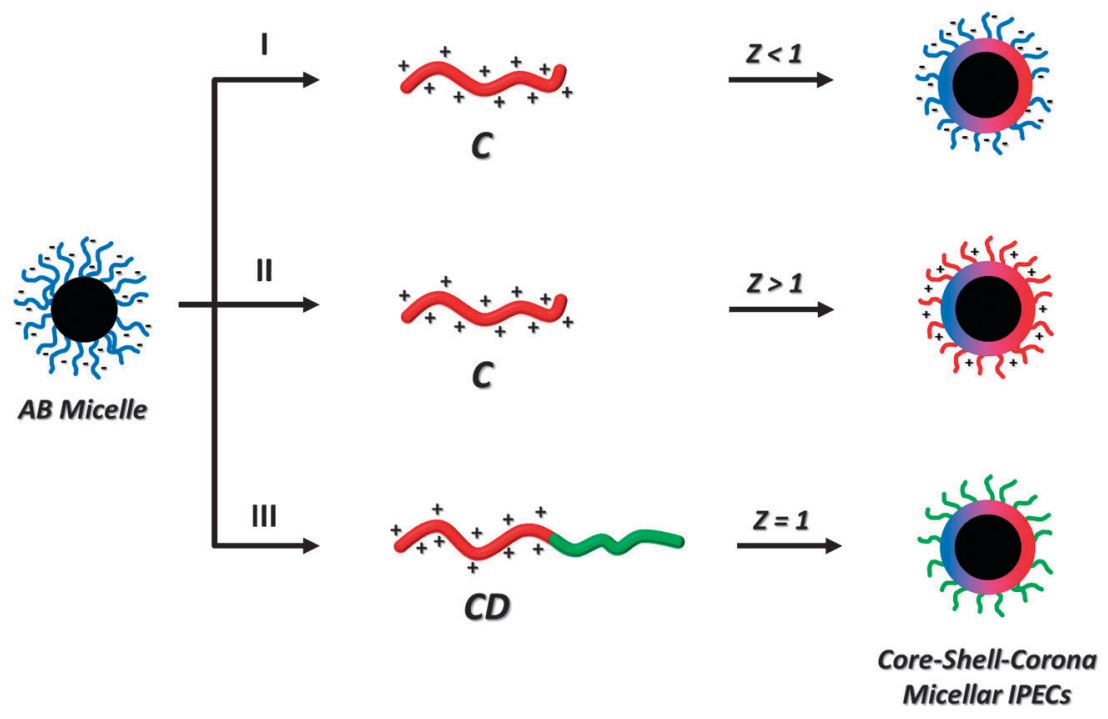

Fig. 6 Schematic depiction of the formation of micellar structures with an IPEC shell between micelles from AB diblock copolymers with a negatively charged corona and linear homopolyelectrolytes of opposite charge (C, I + II) or bis-hydrophilic diblock copolymers with a charged (C) and an uncharged hydrophilic segment (D, III). 
sufficient to stabilize the particles, whereas at higher $Z$-values precipitation occurs. On the other hand, if a considerable excess of the oppositely charged homopolyelectrolyte is used, overcharging and, hence, charge inversion for the micellar IPECs can occur as well (II).

If, on the other hand, bis-hydrophilic diblock copolymers (CD) with a charged (C) and an uncharged but hydrophilic segment (D) are used, the generated micellar IPECs are expected to be well soluble even at stoichiometric charge ratios $(Z=1)$. The uncharged hydrophilic D segment builds up a new micellar corona, whereas both $\mathrm{B}$ and $\mathrm{C}$ are buried within the IPEC shell.

\section{$\mathbf{A B}+\mathbf{C}$}

The formation of an IPEC shell was first demonstrated by Talingting et al. for the addition of a considerable excess of PSSNa to micellar solutions of PS- $b$-P2VP at low $\mathrm{pH}$-values $(\mathrm{pH}<1)$, where the P2VP corona of the micelles is fully charged. ${ }^{43}$ Similar results were obtained later by Kabanov and coworkers. Here, PS- $b$-P4VPq block copolymers were used for micelle formation, thus providing particles with a cationic corona even at high $\mathrm{pH}$. Mixing with different amounts of PMANa leads to core-shell-corona architectures with a PS core, an IPEC shell consisting of $\mathrm{P} 4 \mathrm{VPq} / \mathrm{PMANa}$, and a corona formed either by excess P2VPq or PMANa, depending on the actual $Z$-ratio. ${ }^{45}$ At low loadings of PMANa $(Z=[-] /[+]<0.3)$, complex particles with a cationic net charge ( $\mathrm{P} 4 \mathrm{VPq}$ corona) were obtained. Intermediate amounts of PMANa $(0.3 \leq Z=[-] /[+] \leq 1.8)$ lead to precipitation, whereas complex particles with an anionic net charge (PMANa corona) were found at $Z=[-] /[+]>1.8$, due to overcharging effects.

Whereas the aforementioned examples employed a rather rigid (high $T_{\mathrm{g}}$ ) core-forming block (PS), also studies on ionic amphiphilic systems with dynamic (low $T_{\mathrm{g}}$ ) cores have been carried out. For example, polyisobutylene-block-PMANa (PIB-b-PMANa) diblock copolymers undergo self-assembly in aqueous media into micelles with a negatively charged corona at $\mathrm{pH}$-values where PMAA is deprotonated $\left(\mathrm{p} K_{\mathrm{a}}=5.5\right){ }^{64,65}$ The aggregation numbers of such micelles were shown to depend both on the $\mathrm{pH}$ and the salt concentration, thereby manifesting their dynamic nature. Upon the addition of positively charged P4VPq, an IPEC shell (PMANa/P4VPq) is formed while the structures remain water-soluble below a threshold $Z$-value $(Z=[+] /[-])$. The formation of these micellar IPECs has been thoroughly studied by small-angle neutron scattering (SANS) and it was found that aggregation numbers of original PIB- $b$-PMANa micelles do not change upon their complexation with P4VPq. ${ }^{65,66}$ They thus were able to act as peculiar macromolecular templates for the buildup of core-shell-corona architectures. At the same time, IPEC formation did not render "dynamic" PIB- $b$-PMANa micelles "frozen" structures as their aggregation numbers remained sensitive to variations of their environment, e.g., changes in $\mathrm{pH}$ or ionic strength. ${ }^{65,66}$ Further, an increase of the salinity above $0.2 \mathrm{M} \mathrm{NaCl}$ was demonstrated to result in progressive dissociation of the IPEC domains, whereby at values higher than $0.3 \mathrm{M} \mathrm{NaCl}$ a gradual release of $\mathrm{P} 4 \mathrm{VPq}$ chains could be shown by AUC measurements. ${ }^{66}$

\section{ABC}

As has been already shown for linear ABC triblock terpolymers comprising oppositely charged blocks within one single macromolecule, the formation of intra-molecular IPECs is also possible. ${ }^{60}$ In terms of a micellar im-IPEC shell, two systems have been recently introduced by our groups (Fig. 7). In the case of polyampholytic polybutadiene-block-poly(1-methyl-2-vinyl pyridinium iodide)-block-PMAA (PB- $b$-P2VPq- $b$-PMAA) triblock terpolymers, multicompartment micelles with a $\mathrm{PB}$ core, an im-IPEC shell formed via complexation of $\mathrm{P} 2 \mathrm{VPq}$ and PMAA, and a corona of PMAA (if DP $\mathrm{PMAA}_{\mathrm{P}}>\mathrm{DP}_{\mathrm{P} 2 \mathrm{VPq}}$, DP corresponds to the degree of polymerization of the respective segment) were observed. The im-IPEC shell was shown to be patchy, most probably due to the high interfacial energy between $\mathrm{PB}$ and the PMAA/P2VPq compartments, thereby generating well-defined micelles with a "raspberry" morphology (Fig. 7A) ${ }^{67}$ In an attempt to invert the charge of such im-IPEC species, the synthesis and self-assembly of polyampholytic PB- $b$-PMAA- $b$-PDMAEMAq triblock terpolymers has been studied. Here, again multicompartment micelles were formed in aqueous media, exhibiting a PB core of similar size and, depending on the solution $\mathrm{pH}$, an im-IPEC shell of the coupled PMAA/PDMAEMAq, surrounded by a corona formed by excess cationic PDMAEMAq segments (if DP PDMAEMAq $>$ $\mathrm{DP}_{\text {PMAA }}$, Fig. 7B). ${ }^{68}$ In both cases, the micelles were shown to undergo changes in core size (aggregation number) in response to changes in the surrounding conditions (salinity, $\mathrm{pH}$ ) as revealed by cryo-TEM and light scattering experiments. Whereas in the case of PB- $b$-P2VPq-b-PMAA rather high salt concentrations were necessary, micelles formed by PB- $b$-PMAA- $b$-PDMAEMAq were already able to respond to changes in $\mathrm{pH}$. This has been tentatively attributed to a lower density of the im-IPEC shell and, in addition, to a less unfavorable interface between the micellar core $(\mathrm{PB})$ and the IPEC domains formed by PMAA/PDMAEMAq. In comparison, no effect of the $\mathrm{pH}$ was observed for systems with an im-IPEC shell of P2VPq/PMAA. If monolayers of such PB- $b$-PMAA- $b$-PDMAEMAq micelles were immobilized on silica substrates from dilute solution, a dynamic behavior of the particles upon $\mathrm{pH}$-changes could be seen by scanning force microscopy (SFM), resulting in stimuli-responsive, laterally structured surfaces. ${ }^{69}$ The same im-IPEC micelles have also been adsorbed onto metal sponges and these structures then exhibited $\mathrm{pH}$-dependent charge inversion and could be used for the reversible attachment/detachment of cells. ${ }^{70}$

The formation of cylindrical micelles with an im-IPEC shell has been realized via bulk-templating. Here, the $\mathrm{PB}$ domains of a cylindrical bulk morphology of PB- $b$-P2VP- $b-\mathrm{P} t \mathrm{BMA}$ triblock terpolymers were crosslinked using a UV-photoinitiator and subsequent sonication-assisted dissolution in combination with polymer-analogue reactions which yielded core-crosslinked micelles with a PB core, an im-IPEC shell of P2VPq/PMAA, and a PMAA corona (Fig. $7 \mathrm{C})^{71}$

\section{$\mathbf{A B}+\mathbf{C D}$}

If bis-hydrophilic diblock copolymers comprising a charged (C) and an uncharged (D) segment are mixed with oppositely charged micelles $(\mathrm{AB})$ at equimolar charge ratio $(Z=1)$, 


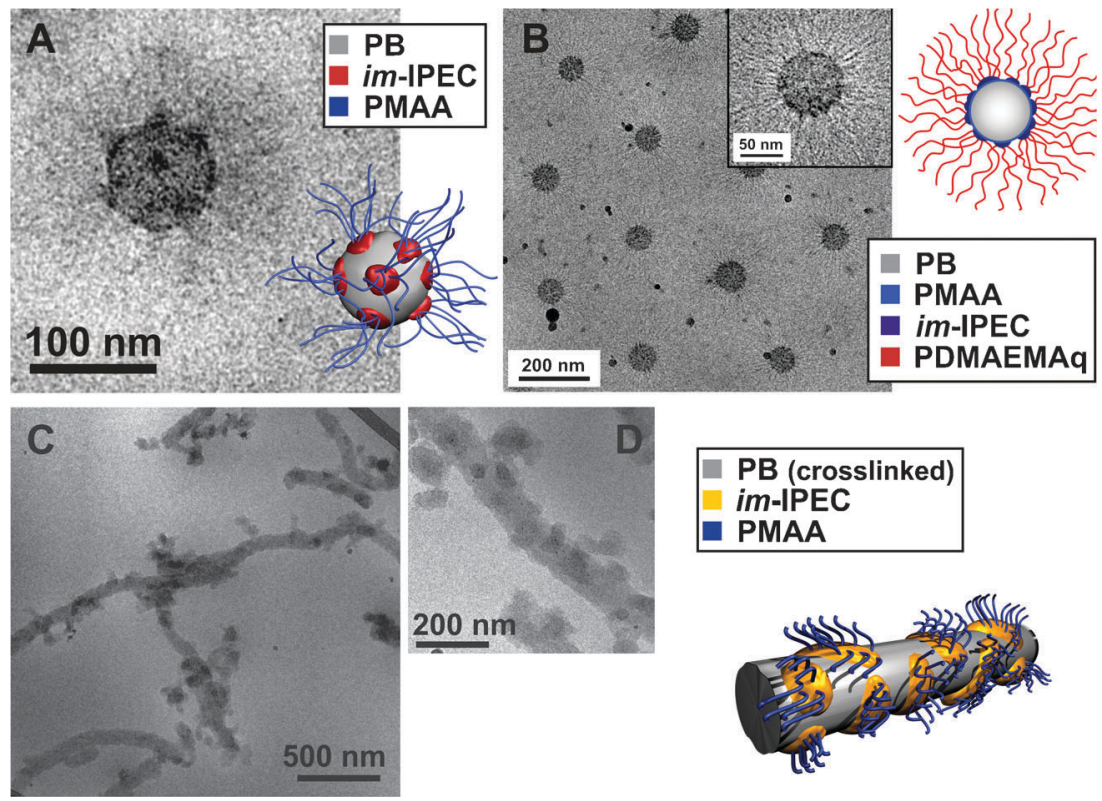

Fig. 7 Different systems comprising im-IPEC shells; cryo-TEM micrographs and schematic depiction of multicompartment micelles from ampholytic PB-b-P2VPq- $b$-PMAA triblock terpolymers (A, reproduced from ref. 67. Copyright 2009 American Chemical Society); comparable structures formed by PB- $b$-PMAA- $b$-PDMAEMAq triblock terpolymers with a cationic corona (B, reproduced from ref. 68. Copyright 2011 Royal Society of Chemistry); core-crosslinked cylindrical micelles prepared via bulk templating from PB- $b$-P2VPq- $b$-PMAA triblock terpolymers (C, reproduced from ref. 71. Copyright 2011 Royal Society of Chemistry).

an IPEC shell is formed immediately and the uncharged D segments build up the corona of the generated core-shellcorona structures (Fig. 6III). The fact that the IPEC shell is then more or less shielded by the D corona chains renders such approaches interesting for further application in, e.g., encapsulation and delivery (see also the last chapter). In that way, mixing of poly(n-butyl acrylate)-block-poly(sodium 2-acrylamido-2-methylpropanesulfonate) (PnBA-b-PAMPS) diblock copolymer micelles with bis-hydrophilic poly(oligoethylene glycol methacrylate)-block-PDMAEMAq (POEGMA$b$-PDMAEMAq) resulted in the formation of multi-layered particles with a $\mathrm{P} n \mathrm{BA}$ core, an IPEC shell of PAMPS/ PDMAEMAq, and a POEGMA corona. ${ }^{72}$ A similar strategy has been pursued by Cametti et al.: here, the hydrophobic core was formed by the PNIPAAm segments of PAMPS- $b$ PNIPAAm diblock copolymers above the LCST and complexation with oppositely charged PEO- $b$-poly(3-acrylamidopropyl trimethylammonium chloride) (PEO- $b$-PAMPTMA) yielded micelles with a PAMPS/PAMPTMA shell. The presence of this IPEC shell prevented the dissolution of the PNIPAAm core even below the LCST temperature, yielding coreshell-corona structure where the core could be reversibly swollen/contracted. ${ }^{73}$

Armes and coworkers have used a comparable approach (crosslinking via an IPEC shell) for a combination of PEO$b$-PDMAEMAq- $b$-PDEAEMA triblock terpolymers and PEO- $b$-PSSNa diblock copolymers. ${ }^{74}$ At high $\mathrm{pH}$, PEO- $b$ PDMAEMAq- $b$-PDEAEMA forms micelles with a hydrophobic PDEAEMA core, a cationic PDMAEMAq shell, and a PEO corona. If now PEO- $b$-PSSNa is added, an IPEC shell of PDMAEMAq/PSSNa is formed and the corona consists of PEO segments from both polymeric materials. The IPEC shell serves as a peculiar crosslinker to stabilize the PDEAEMA core even under conditions where PDEAEMA would be well soluble in aqueous media, i.e. at low pH. The IPEC shell in this particular case remained intact up to $\mathrm{NaCl}$ concentrations of $1.0 \mathrm{M}$.

\section{Advanced structures comprising IPEC domains}

After its discovery in $1997,{ }^{3}$ the layer-by-layer approach could also be transferred to colloidal templates, as shown by Caruso and Möhwald. ${ }^{31}$ Here, sequential electrostatic assembly of positively charged PDADMAC and negatively charged $\mathrm{SiO}_{2}$ nanoparticles onto PS latex particles resulted in the formation of hybrid multilayer-coated particles, which, after calcination, could be transformed into hollow silica spheres. In general, layer-by-layer approaches have been widely used for the preparation of stimuli-responsive capsules or to develop nanometer-sized protective shells for encapsulated cargo in drug-delivery applications. For more details, the reader is referred to a very recent review. ${ }^{75}$

Our groups recently demonstrated a facile approach for the preparation of multi-layered micellar IPECs: starting from core-shell-corona micelles with a $\mathrm{PB}$ core, a discontinuous im-IPEC shell of P2VPq/PMAA, and a negatively charged corona made up of an excess of PMAA, a second IPEC shell could be generated through the addition of bis-hydrophilic PEO- $b$-P2VPq diblock copolymers. ${ }^{76}$ The particles were stabilized through the newly formed PEO corona. In this case both IPEC shells had the same composition (P2VPq/PMAA) and thus could not be distinguished in cryo-TEM micrographs. Using a different bis-hydrophilic diblock copolymer, PEO- $b$-PDMAEMAq, and the same PB- $b$-P2VPq- $b$-PMAA precursor micelles, double-layered micelles with two distinct IPEC layers (1st P2VPq/PMAA, 2nd PMAA/PDMAEMAq) 

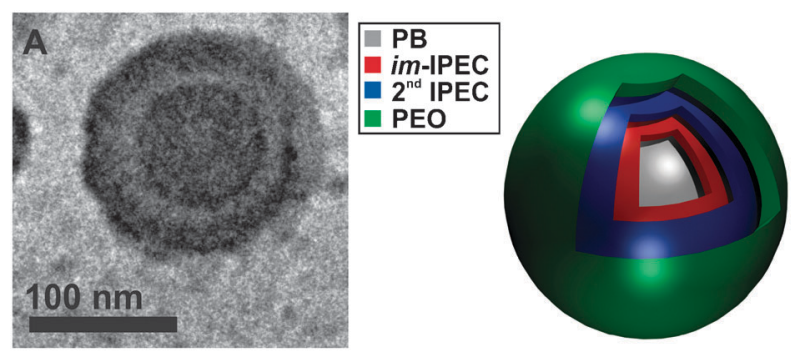

B

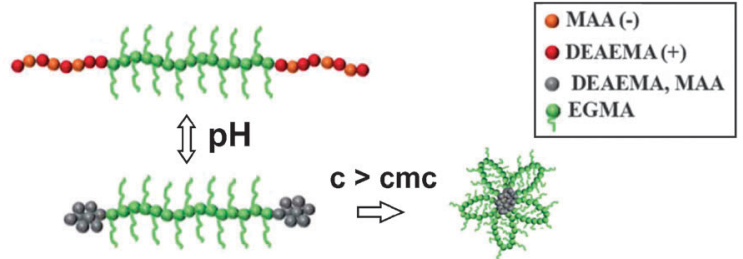

Fig. 8 Cryo-TEM micrograph of multi-layered micellar IPECs prepared via the mixing of PB- $b$-P2VPq- $b$-PMAA triblock terpolymer micelles and bis-hydrophilic PEO- $b$-PDMAEMAq diblock copolymers (A, reproduced from ref. 77. Copyright 2011 Royal Society of Chemistry); $\mathrm{pH}$ - and concentration-dependent association of gradient polyampholytic (PMAA-co-PDEAEMA)- $b$-POEGMA- $b$-(PMAA-coPDEAEMA) graft copolymers (B, reproduced from ref. 78. Copyright 2011 Royal Society of Chemistry).

could be prepared (Fig. 8A). ${ }^{77}$ In cryo-TEM experiments, five different domains within these structures were also identified: the PB core, the 1st IPEC shell, a transition layer of highly swollen PMAA, the 2nd IPEC shell, and, finally, the PEO corona. For both systems, intermediate non-equilibrium structures with either ray-like protrusions ${ }^{76}$ or an inhomogeneous distribution of guest polyelectrolyte segments among the precursor micelles were observed. ${ }^{77}$

In another example of intra-molecular IPEC formation, Tsitsilianis et al. investigated ampholytic P(DEAEMA-co-MAA)$b$-poly(oligoethylene glycol methacrylate)- $b$-P(DEMAEMA$c o$-MAA) triblock terpolymers (P(DEAEMA-co-MAA)- $b$ POEGMA- $b$-P(DEMAEMA-co-MAA)). ${ }^{78}$ At intermediate $\mathrm{pH}$ values, IPEC formation occurs between positively charged PDEAEMA and negatively charged PMAA, leading to the formation of complex satellites, stabilized by the hydrophilic POEGMA segments. At sufficiently high concentration, these further aggregated into flower-like superstructures with PDEAEMA/PMAA cores (Fig. 8B).

As an example for 3D network structures via IPEC formation in more concentrated solutions, the $\mathrm{pH}$ - and concentration-dependent formation of physical gels from ampholytic PAA- $b$-P2VP-b-PAA triblock copolymers has been investigated by Tsitsilianis et al. ${ }^{79}$ Multi-responsive gels could be realized via the combination of double hydrophilic, negatively charged poly(sulfopropyl methylacrylate)-block-PEO-blockpoly(sulfopropyl methacrylate) (PSPMA- $b$-PEO- $b$-PSPMA) triblock copolymers and positively charged poly(allylammonium hydrochloride) (PAH) homopolymers. ${ }^{80}$ The network properties of the resulting structures could be tuned by several parameters, including $\mathrm{pH}$, temperature, and the chargeto-charge stoichiometry. In a comparable recent approach, complex coacervate hydrogels have been prepared by the groups of Kramer and Hawker. ${ }^{81}$ Here, ABA triblock copolymers of poly(allyl glycidyl ether)-block-PEO-blockpoly(allyl glycidyl ether) (PAGE- $b$-PEO- $b$-PAGE) were modified using thiol-ene chemistry so that the A segments carried either cationic or anionic moieties. If two oppositely charged $\mathrm{ABA} / \mathrm{A}^{\prime} \mathrm{BA}^{\prime}$ systems were mixed at concentrations of about $10 \mathrm{wt} \%$ in aqueous media of different $\mathrm{pH}$ values, transparent hydrogels were formed by complexation between $\mathrm{A}$ and $\mathrm{A}^{\prime}$ building blocks.

\section{IPECs in organic solvents}

Micelles with an IPEC core can also be prepared in organic solvents of (typically) low polarity. As an example, the mixing of non-equivalent amounts of the cetyltrimethylammonium (CTMA) salt of PS- $b$-PAA block copolymers (PS- $b$-PA ${ }^{-}$ $\mathrm{CTMA}^{+}$) and the dodecyl sulfate (DDS) salt of PDMAEMAq $\left(\mathrm{PDMAEMA}^{+} \mathrm{DDS}^{-}\right.$) in chloroform leads to the release of the surfactant counterions and the formation of micellar IPECs. Those structures exhibited a mixed corona that was formed by PS chains and, depending on which of the two ionic segments has been taken in excess, either PDMAEMA ${ }^{+}$ DDS $^{-}$or $\mathrm{PA}^{-} \mathrm{CTMA}^{+}$segments. ${ }^{82}$ Schlaad and coworkers reported on vesicles (polymersomes) with an IPEC membrane wall by mixing two ionic amphiphilic block copolymers, PB-b-poly(cesium methacrylate) (PB-b-PMACs) and PS- $b$-P4VPq, in a polar organic solvent (THF), where the individual block copolymers form reverse micelles. ${ }^{83}$ Remarkably, the IPEC vesicles were asymmetric in nature: the inside of the membrane wall was covered with the PB segments while the PS segments were located on the outside of the polymersomes.

\section{Possible applications (especially for micellar IPECs)}

The possibility to control the extent of interpolyelectrolyte complexation and the location of the IPEC domains within micellar structures renders this class of materials very interesting for applications where the controlled positioning of an interface, the encapsulation of a specific "payload", the generation of charges, or simply the attachment of certain functionalities is crucial. Certainly, one clear focus will be the use of micellar structures with IPEC cores or shells as biocompatible carriers in drug delivery applications. Several advantages of such systems can be identified:

(I) Interpolyelectrolyte complex formation offers a simple and efficient approach for the encapsulation of ionic biopharmaceuticals, especially DNA or RNA and proteins, within selected domains of micellar structures. ${ }^{40,84}$

(II) Changes in both $\mathrm{pH}$ and ionic strength of the surrounding environment are capable of inducing the dissociation of IPECs and represent straightforward methodologies for the release of encapsulated cargo at target. ${ }^{40}$

(III) The possibility to equip such micellar IPECs with a biocompatible and protective (optionally stimuli-responsive, e.g., thermosensitive) corona of, e.g., PEO or PNIPAAm, in a one-step procedure through the use of bis-hydrophilic PEO- $b$ polyion block copolymers. ${ }^{85}$

(IV) Constant progress in controlled/living polymerization techniques or post-polymerization modifications enables superior control over both the location and the density of charges 
within polymeric materials; this is accompanied by the preparation, functionalization, or the "labeling" of block copolymers comprising polyionic segments.

Another intriguing feature of micellar architectures bearing charges and/or having specifically located IPEC domains is that they can be employed for the controlled selective deposition of metal nanoparticle (NP) precursors, leading to the corresponding NPs after reduction. In that way, the formation of well-defined inorganic-organic hybrid materials becomes feasible. As one example, this has been demonstrated for multi-layered micellar IPECs based on PB- $b$-P2VPq- $b$ PMAA triblock terpolymers complexed with PEO- $b-\mathrm{P} 2 \mathrm{VPq}$ diblock copolymers, where small-sized Au-NPs $(\sim 2 \mathrm{~nm})$ were exclusively located within the IPEC shell formed from P2VPq/PMAA segments. ${ }^{76}$ Further, Pd-NPs were solely generated within the patchy im-IPEC shell of core-crosslinked cylinders from PB- $b$-P2VPq- $b$-PMAA triblock terpolymers, ${ }^{71}$ although the exact reason for this phenomenon is still not fully understood.

Another possibility is the use of IPEC formation for the adjustment of, e.g., mechanical properties within hierarchically structured composite materials. This has been demonstrated for the electrostatic co-assembly of cellulose nanofibers with an anionic surface charge and cationic PB- $b$-PDMAEMAq micelles. ${ }^{86}$ The electrostatic attraction between the stiff cellulose nanofibers and the flexible polymeric building blocks prevented macroscopic phase separation and allowed for a true alternating hard/soft architecture of the materials. This can offer further perspectives for the development of novel generations of composite materials with unique characteristics and properties, which cannot be realized by conventional blending techniques.

\section{Future perspective}

There clearly are some open questions regarding the formation or the stability of micellar IPECs. For example, in some cases complete dissociation of the IPEC domains requires rather high salt concentrations, as shown for the im-IPEC domains in multi-compartment micelles $(>0.5 \mathrm{M} \mathrm{NaCl})^{67}$ or the IPEC shell on PIB- $b$-PMANa structures $(c a .0 .5 \mathrm{M} \mathrm{NaCl}) .{ }^{66}$ In other cases, changes in $\mathrm{pH}$ were already sufficient to facilitate rearrangements within IPEC domains. ${ }^{68}$ It has been reported, though, that the strength of the ionic binding may well depend on the nature of the ionic groups or the respective counterions. ${ }^{25,49-51}$ Nevertheless, the question remains whether it is possible to pre-estimate the strength (with respect to dissociation) of an IPEC domain according to the constituting polyelectrolytes, e.g., the local charge density or the distance of the ionic group from the polymeric backbone. This might allow polymer chemists in the near future to prepare micelles with IPEC cores (or shells), which provide an exact "release point". The latter could then be adjusted to the targeted environment, i.e., $\mathrm{pH}$ or salinity within a narrow regime.

Concerning encapsulation/delivery applications in general, it would be of interest to incorporate more than one "payload" within micellar IPECs. The development of multi-layered IPEC structures may provide a valuable tool to realize this, both in micellar structures with a few layers only or in truly multi-layered (hybrid) capsules derived from layer-by-layer processes. A sequential buildup of concentric layers of (ideally) different chemical functionality might be exploited for (also) sequential encapsulation of a series of guest substances. If those are released afterwards, the question arises whether this release is sequence-controlled (inverse order of loading) or merely dominated by diffusion, leading to a mixture of the individual components. The latter might be partially influenced by differences in solvophilicity/solvophobicity of the guest molecules.

Another (closely related) issue is whether the concentric multi-layered architecture of micellar IPECs could be extended to analogous hybrid structures. Similar to established templating approaches where the formation of silica capsules was demonstrated, ${ }^{31}$ the controlled deposition of, e.g., Au and Pd-NPs in consecutive IPEC layers of micellar structures could lead to layered metal or metal-alloy structures. This though implies rather high loading efficiencies of the IPEC domains, which might be difficult to realize using calcination for the removal of the organic part. Low loadings would prevent the formation of continuous metal structures. An alternative approach might be the use of degradable polyelectrolytes such as polyesters with ionizable groups located in the side-chain. Overall, such inorganic/organic hybrid materials could also be considered as prototypes of novel nanoreactors.

Finally, within several studies intermediate or non-equilibrium structures during IPEC formation (and relaxation) could be identified (Fig. 9). Although it has been shown that the
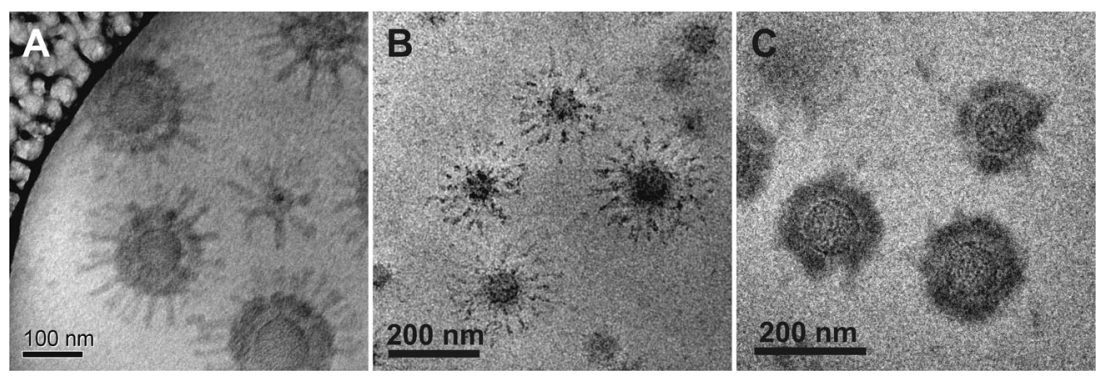

Fig. 9 Cryo-TEM micrographs of intermediate structures during IPEC formation between PB- $b$-P2VPq- $b$-PMAA triblock terpolymers and PEO- $b$-P2VPq diblock copolymers at $Z=1$ (A, reproduced from ref. 76. Copyright 2009 American Chemical Society); after mixing of PB- $b$-P2VPq- $b$-PMAA/PEO- $b$-P2VPq core-shell-corona IPECs $(Z=1)$ with PB- $b$-P2VPq- $b$-PMAA triblock terpolymer micelles (B, unpublished data); during the formation of multi-layered micellar IPECs from PB- $b$-P2VPq- $b$-PMAA/PEO- $b$-PDMAEMAq at $Z=[+] /[-]=0.25$ (C, reproduced from ref. 77. Copyright 2011 Royal Society of Chemistry). 
formation of equilibrium IPECs in dilute solution occurs within several milliseconds, ${ }^{41}$ intermediate structures exhibiting ray-like protrusions could be identified after mixing of PB- $b$-P2VPq- $b$-PMAA triblock terpolymer micelles with oppositely charged PEO- $b$-P2 VPq diblock terpolymers at $Z=1$ (Fig. 9A). ${ }^{76}$ This was tentatively attributed to steric crowding of the block copolymer chains during the initial complexation, followed by relaxation into core-shell-corona equilibrium structures within several days. If these micellar IPECs were then mixed with "native" PB- $b$-P2VPq- $b$-PMAA precursor micelles so that the overall $Z=[+] /[-]=0.5$, intermediate structures of different morphology were observed (Fig. 9B), thereby providing clear evidence on polyion exchange reactions occurring for this system. If, on the other hand, the formation of the 2nd IPEC shell was carried out at rather low $Z$-values $(Z=[+] /[-]=0.25$, PB- $b$-P2VPq- $b$ PMAA/PEO- $b$-PDMAEMAq), mixtures of micelles at different states of complexation were found in cryo-TEM (Fig. 9C). ${ }^{77}$ All these findings do suggest that not all processes during IPEC formation, at least for micellar structures, are currently fully understood. It rather implies that additional factors like steric crowding (at the IPEC/non-IPEC interface), especially the volume of the uncharged block (for bis-hydrophilic block copolymers), which is not involved in IPEC formation but incompatible with the IPEC domains, have to be taken into account very carefully.

Nevertheless, the electrostatic co-assembly of polymeric building blocks into micellar IPECs offers unique potential for the fabrication of well-defined, hierarchically structured materials on the nanoscale. A unique variety of polymeric materials, which can be involved in IPEC formation, provides an attractive opportunity to manipulate micellar characteristics in a desired manner. Processes that offer such levels of control over dimension, architecture, functionality, and charge of nanometer-sized domains are scarce and the potential to use IPEC-based materials in life science, sensing, delivery, or the bottom-up structuring of functional materials is huge.

\section{Abbreviations}

$\begin{array}{ll}\text { AUC } & \text { analytical ultracentrifugation } \\ \text { BIC } & \text { block ionomer complex } \\ \text { CC } & \text { complex coacervate } \\ \text { CTMA } & \text { cetyltrimethylammonium } \\ \text { DDS } & \text { dodecyl sulfate } \\ \text { DNA } & \text { deoxyribonucleic acid } \\ \text { DP } & \text { degree of polymerization } \\ \text { im-IPEC } & \text { intra-micellar interpolyelectrolyte complex } \\ \text { IPEC } & \text { interpolyelectrolyte complex } \\ \text { NP } & \text { nanoparticle } \\ \text { OPs } & \text { oligophosphates } \\ \text { P2VP } & \text { poly(2-vinyl pyridine) } \\ \text { P4VP } & \text { poly(4-vinyl pyridine) } \\ \text { PAA } & \text { poly(acrylic acid) } \\ \text { PAAm } & \text { polyacrylamide } \\ \text { PAGE } & \text { poly(allyl glycidyl ether) } \\ \text { PAH } & \text { poly(allylammonium hydrochloride) } \\ \text { PAMPS } & \text { poly(sodium 2-acrylamido- } \\ & \text { 2-methylpropanesulfonate) }\end{array}$

PAMPTMA poly(3-acrylamidopropyl trimethylammonium chloride)

PAsp poly(aspartic acid)

PB polybutadiene

PDADMAC poly(diallyldimethylammonium chloride)

PDEAEMA poly(2-(diethylamino)ethyl methacrylate)

PDMAEMA poly(2-(dimethylamino)ethyl methacrylate)

PEO

PGMA poly(glycidyl methacrylate)

PIB polyisobutylene

PIC polyion complex

PIL poly(ionic liquid)

PLL poly(L-lysine)

PLys polylysine

PMAA poly(methacrylic acid)

PMACs poly(cesium methacrylate)

PMANa poly(sodium methacrylate)

PnBA poly(n-butyl acrylate)

PNiPAAm poly( $N$-isopropyl acrylamide $)$

POEGMA poly(oligoethylene glycol methacrylate)

PPs polyphosphates

PS polystyrene

PSEMA poly(2-(succinyloxy)ethyl methacrylate)

PSPMA poly(sulfopropyl methacrylate)

PSSNa poly(styrenesulfonic acid sodium salt)

RNA ribonucleic acid

SANS small angle neutron scattering

SFM scanning force microscopy

TEM transmission electron microscopy

THF tetrahydrofuran

\section{Acknowledgements}

D. V. P. acknowledges the Deutsche Forschungsgemeinschaft (DFG) and the Sonderforschungsbereich "Von partikulären Nanosystemen zur Mesotechnologie" (SFB 840) for financial support of his research stays at Universität Bayreuth. A. H. E. M. and F. H. S. are grateful to the Volkswagen Foundation for financial support within the framework "Complex Materials". F. H. S. further thanks the DAAD for a postdoctoral and the Verband der chemischen Industrie (VCI) for a starting independent researcher fellowship. He also thanks the Thuringian Ministry for Education, Science, and Culture (TMBWK; grants \#B514-09051, NanoConSens, and \#B515-10065, ChaPoNano). In addition, F. H. S. is indebted to the CSG e.V. for continuous support.

\section{References}

1 R. M. Fuoss and H. Sadek, Science, 1949, 110, 552-554.

2 A. S. Michaels and R. G. Miekka, J. Phys. Chem., 1961, 65, 1765-1773.

3 G. Decher, Science, 1997, 277, 1232-1237.

4 J. van der Gucht, E. Spruijt, M. Lemmers and M. A. Cohen Stuart, J. Colloid Interface Sci., 2011, 361, 407-422.

5 D. V. Pergushov, O. V. Borisov, A. B. Zezin and A. H. E. Müller, Adv. Polym. Sci., 2011, 241, 131-161.

6 A. Jensen, F. Basolo and H. M. Neumann, J. Am. Chem. Soc., 1959, 81, 509-512.

7 J. E. Boothe, H. G. Flock and M. F. Hoover, J. Macromol. Sci., Chem., 1970, 4, 1419-1430.

8 E. M. Loebl and J. J. O’Neill, J. Polym. Sci., 1960, 45, 538-540. 
9 V. Bütün, N. C. Billingham and S. P. Armes, Chem. Commun., 1997, 671-672.

10 F. A. Plamper, A. Schmalz, E. Penott-Chang, M. Drechsler, A. Jusufi, M. Ballauff and A. H. E. Müller, Macromolecules, 2007, 40, 5689-5697.

11 C. Sawaryn, K. Landfester and A. Taden, Macromolecules, 2011, 44, 7668-7674.

12 A. Laschewsky, Curr. Opin. Colloid Interface Sci., 2012, 17, 56-63.

13 A. S. Shaplov, E. I. Lozinskaya, R. Losada, C. Wandrey, A. T. Zdvizhkov, A. A. Korlyukov, K. A. Lyssenko, I. A. Malyshkina and Y. S. Vygodskii, Polym. Adv. Technol., 2011, 22, 448-457.

14 J. Yuan and M. Antonietti, Polymer, 2011, 52, 1469-1482.

15 D. Mecerreyes, Prog. Polym. Sci., 2011, 36, 1629-1648.

16 A. Ciferri and S. Kudaibergenov, Macromol. Rapid Commun., 2007, 28, 1953-1968.

17 A. B. Lowe and C. L. McCormick, Chem. Rev., 2002, 102, 4177-4190.

18 A. V. Kabanov and A. B. Zezin, Pure Appl. Chem., 1984, 56, 343-357.

19 B. Philipp, H. Dautzenberg, K.-J. Linow, J. Kötz and W. Dawydoff, Prog. Polym. Sci., 1989, 14, 91-172.

20 A. F. Thünemann, M. Müller, H. Dautzenberg, J.-F. Joanny and H. Löwen, Adv. Polym. Sci., 2004, 166, 113-171.

21 F. W. Tiebackx, Z. Chem. Ind. Kolloide, 1911, 8, 198-201.

22 H. G. Bungenberg de Jong and H. R. Kruyt, Proc. K. Ned. Akad. Wet., 1929, 32, 849-856.

23 E. Tsuchida, Y. Osada and K. Sanada, J. Polym. Sci., Part A: Polym. Chem., 1972, 10, 3397-3404.

24 E. Tsuchida, K. Abe and M. Honma, Macromolecules, 1976, 9, $112-117$.

25 V. A. Kabanov, Russ. Chem. Rev., 2005, 74, 3-20.

26 A. B. Zezin, V. A. Izumrudov and V. A. Kabanov, Makromol. Chem., Macromol. Symp., 1989, 26, 249-264.

27 A. V. Kabanov, T. K. Bronich, V. A. Kabanov, K. Yu and A. Eisenberg, Macromolecules, 1996, 29, 6797-6802.

28 A. Nakajima and H. Sato, Bull. Inst. Chem. Res., Kyoto Univ., 1969, 47, 177-183.

29 M. A. Cohen Stuart, N. A. M. Besseling and R. G. Fokkink, Langmuir, 1998, 14, 6846-6849.

30 V. A. Kabanov, A. B. Zezin, V. A. Kasaikin, A. A. Yaroslavov and D. A. Topchiev, Russ. Chem. Rev., 1991, 60, 288-291.

31 F. Caruso, R. A. Caruso and H. Möhwald, Science, 1998, 282, 1111-1114.

32 Q. Zhao, Q. F. An, Y. Ji, J. Qian and C. Gao, J. Membr. Sci., 2011, 379, 19-45.

33 A. Harada and K. Kataoka, Science, 1999, 283, 65-67.

34 V. A. Izumrudov, A. B. Zezin and V. A. Kabanov, Russ. Chem. Rev., 1991, 60, 792-806.

35 S. Katayose and K. Kataoka, Bioconjugate Chem., 1997, 8, 702-707.

36 T. K. Bronich, H. K. Nguyen, A. Eisenberg and A. V. Kabanov, J. Am. Chem. Soc., 2000, 122, 8339-8343.

37 A. V. Kabanov and V. A. Kabanov, Bioconjugate Chem., 1995, 6, $7-20$.

38 H. Dautzenberg, N. Karibyants and S. Y. Zaitsev, Macromol. Rapid Commun., 1997, 18, 175-182.

39 A. B. Zezin, B. S. Eltsefon, A. R. Rudman, N. A. Vengerova, R. I. Kalyuzhnaya, S. P. Valueva, E. M. Kopylova, A. K. Chepurov, V. S. Efimov and V. A. Kabanov, Pharm. Chem. J., 1987, 21, 464-477.

40 Y. Lee and K. Kataoka, Soft Matter, 2009, 5, 3810-3817.

41 K. N. Bakeev, V. A. Izumrudov, S. I. Kuchanov, A. B. Zezin and V. A. Kabanov, Macromolecules, 1992, 25, 4249-4254.

42 E. K. Penott-Chang, D. V. Pergushov, A. B. Zezin and A. H. E. Müller, Langmuir, 2010, 26, 7813-7818.

43 M. R. Talingting, U. Voigt, P. Munk and S. E. Webber, Macromolecules, 2000, 33, 9612-9619.

44 S. Holappa, L. Kantonen, T. Andersson, F. Winnik and H. Tenhu, Langmuir, 2005, 21, 11431-11438.

45 E. A. Lysenko, P. S. Chelushkin, T. K. Bronich, A. Eisenberg, V. A. Kabanov and A. V. Kabanov, J. Phys. Chem. B, 2004, 108, 12352-12359.
46 A. V. Kharenko, R. I. Kalyuzhnaya, A. B. Zezin and V. A. Kabanov, Vysokomol. Soedin., Ser. A, 1981, 23, 2657-2666.

47 A. Harada and K. Kataoka, Macromolecules, 2003, 36, 4995-5001.

48 D. V. Pergushov, V. A. Izumrudov, A. B. Zezin and V. A. Kabanov, Polym. Sci., Ser. A, 1995, 37, 1081-1087.

49 H. Dautzenberg and N. Karibyants, Macromol. Chem. Phys., 1999, 200, 118-125.

50 D. V. Pergushov, V. A. Izumrudov, A. B. Zezin and V. A. Kabanov, Polym. Sci., 1993, 35, 940-944.

51 H. Dautzenberg and J. Kriz, Langmuir, 2003, 19, 5204-5211.

52 P. S. Chelushkin, E. A. Lysenko, T. K. Bronich, A. Eisenberg, V. A. Kabanov and A. V. Kabanov, J. Phys. Chem. B, 2008, 112, $7732-7738$.

53 Y. Li, T. K. Bronich, P. S. Chelushkin and A. V. Kabanov, Macromolecules, 2008, 41, 5863-5868.

54 Y. Kakizawa, A. Harada and K. Kataoka, J. Am. Chem. Soc., 1999, 121, 11247-11248.

55 A. Harada and K. Kataoka, Macromolecules, 1995, 28, 5294-5299.

56 J.-F. Gohy, S. K. Varshney and R. Jérôme, Macromolecules, 2001, 34, 3361-3366.

57 I. K. Voets, A. de Keizer and M. A. Cohen Stuart, Adv. Colloid Interface Sci., 2009, 147-148, 300-318.

58 I. K. Voets, A. de Keizer, P. de Waard, P. M. Frederik, P. H. H. Bomans, H. Schmalz, A. Walther, S. M. King, F. A. M. Leermakers and M. A. Cohen Stuart, Angew. Chem., Int. Ed., 2006, 45, 6673-6676.

59 I. K. Voets, P. M. Moll, A. Aqil, C. Jérôme, C. Detrembleur, P. d. Waard, A. de Keizer and M. A. C. Stuart, J. Phys. Chem. B, 2008, 112, 10833-10840.

60 Y. Cai and S. P. Armes, Macromolecules, 2004, 37, 7116-7122.

61 H. Liu, C. Li, H. Liu and S. Liu, Langmuir, 2009, 25, 4724-4734.

62 Y. Yan, L. Harnau, N. A. M. Besseling, A. de Keizer, M. Ballauff, S. Rosenfeldt and M. A. Cohen Stuart, Soft Matter, 2008, 4, 2207-2212.

63 G. Riess, Prog. Polym. Sci., 2003, 28, 1107-1170.

64 D. V. Pergushov, E. V. Remizova, J. Feldthusen, A. B. Zezin, A. H. E. Müller and V. A. Kabanov, J. Phys. Chem. B, 2003, 107, 8093-8096.

65 D. V. Pergushov, E. V. Remizova, M. Gradzielski, P. Lindner, J. Feldthusen, A. B. Zezin, A. H. E. Müller and V. A. Kabanov, Polymer, 2004, 45, 367-378.

66 M. Burkhardt, M. Ruppel, S. Tea, M. Drechsler, R. Schweins, D. V. Pergushov, M. Gradzielski, A. B. Zezin and A. H. E. Müller, Langmuir, 2008, 24, 1769-1777.

67 F. Schacher, A. Walther and A. H. E. Müller, Langmuir, 2009, 25, 10962-10969.

68 E. Betthausen, M. Drechsler, M. Förtsch, F. H. Schacher and A. H. E. Müller, Soft Matter, 2011, 7, 8880-8891.

69 J. Gensel, E. Betthausen, C. Hasenöhrl, K. Trenkenschuh, M. Hund, F. Boulmedais, P. Schaaf, A. H. E. Müller and A. Fery, Soft Matter, 2011, 7, 11144-11153.

70 J. Gensel, T. Borke, N. P. Pérez, A. Fery, D. V. Andreeva, E. Betthausen, A. H. E. Müller, H. Möhwald and E. V. Skorb, Adv. Mater., 2012, 24, 985-989.

71 F. H. Schacher, T. Rudolph, M. Drechsler and A. H. E. Müller, Nanoscale, 2011, 3, 288-297.

72 J.-F. Lutz, S. Geffroy, H. von Berlepsch, C. Bottcher, S. Garnier and A. Laschewsky, Soft Matter, 2007, 3, 694-698.

73 G. Masci, S. De Santis and C. Cametti, J. Phys. Chem. B, 2011, 115, 2196-2204.

74 J. V. M. Weaver, Y. Tang, S. Liu, P. D. Iddon, R. Grigg, N. C. Billingham, S. P. Armes, R. Hunter and S. P. Rannard, Angew. Chem., Int. Ed., 2004, 43, 1389-1392.

75 M. Delcea, H. Möhwald and A. G. Skirtach, Adv. Drug Delivery Rev., 2011, 63, 730-747.

76 F. Schacher, E. Betthausen, A. Walther, H. Schmalz, D. V. Pergushov and A. H. E. Müller, ACS Nano, 2009, 3, 2095-2102.

77 C. V. Synatschke, F. H. Schacher, M. Förtsch, M. Drechsler and A. H. E. Müller, Soft Matter, 2011, 7, 1714-1725.

78 Z. Iatridi, G. Mattheolabakis, K. Avgoustakis and C. Tsitsilianis, Soft Matter, 2011, 7, 11160-11168. 
79 F. Bossard, V. Sfika and C. Tsitsilianis, Macromolecules, 2004, 37, 3899-3904.

80 M. Lemmers, J. Sprakel, I. K. Voets, J. van der Gucht and M. A. Cohen Stuart, Angew. Chem., Int. Ed., 2010, 49, 708-711.

81 J. N. Hunt, K. E. Feldman, N. A. Lynd, J. Deek, L. M. Campos, J. M. Spruell, B. M. Hernandez, E. J. Kramer and C. J. Hawker, Adv. Mater., 2011, 23, 2327-2331.

82 E. K. Penott-Chang, M. Ruppel, D. V. Pergushov, A. B. Zezin and A. H. E. Müller, Polymer, 2011, 52, 4296-4302.
83 S. Schrage, R. Sigel and H. Schlaad, Macromolecules, 2003, 36, $1417-1420$.

84 K. T. Oh, T. K. Bronich, L. Bromberg, T. A. Hatton and A. V. Kabanov, J. Controlled Release, 2006, 115, 9-17.

85 N. Bayó-Puxan, M.-H. Dufresne, A. E. Felber, B. Castagner and J.-C. Leroux, J. Controlled Release, 2011, 156, 118-127.

86 M. Wang, A. Olszewska, A. Walther, J.-M. Malho, F. H. Schacher, J. Ruokolainen, M. Ankerfors, J. Laine, L. A. Berglund, M. Österberg and O. Ikkala, Biomacromolecules, 2011, 12, 2074-2081. 